\title{
Particle and VOC emission factor measurements for anthropogenic sources in West Africa
}

\section{Sekou Keita et al.}

Correspondence to: Sekou Keita (sekkeith@yahoo.fr)

The copyright of individual parts of the supplement might differ from the CC BY 4.0 License. 

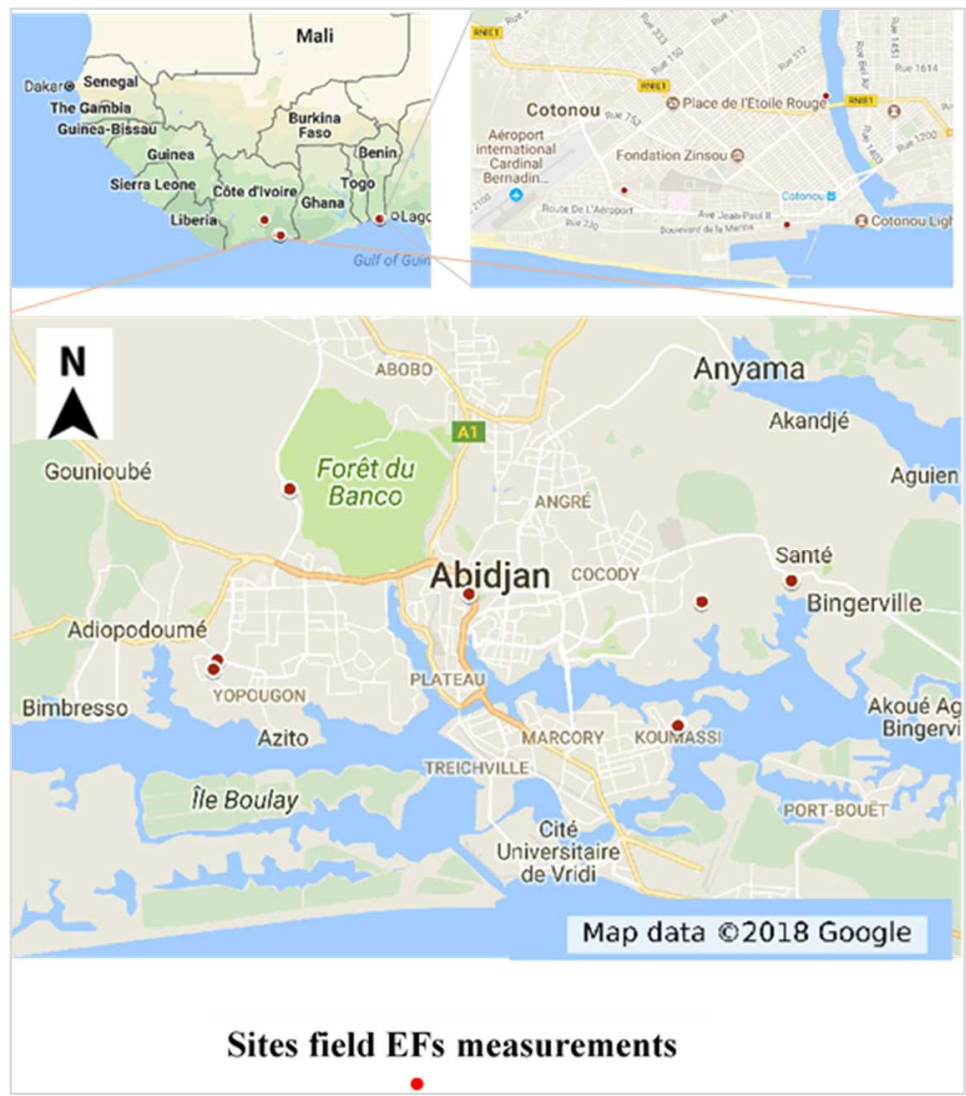

Figure S1: Locations of field EFs measurements 


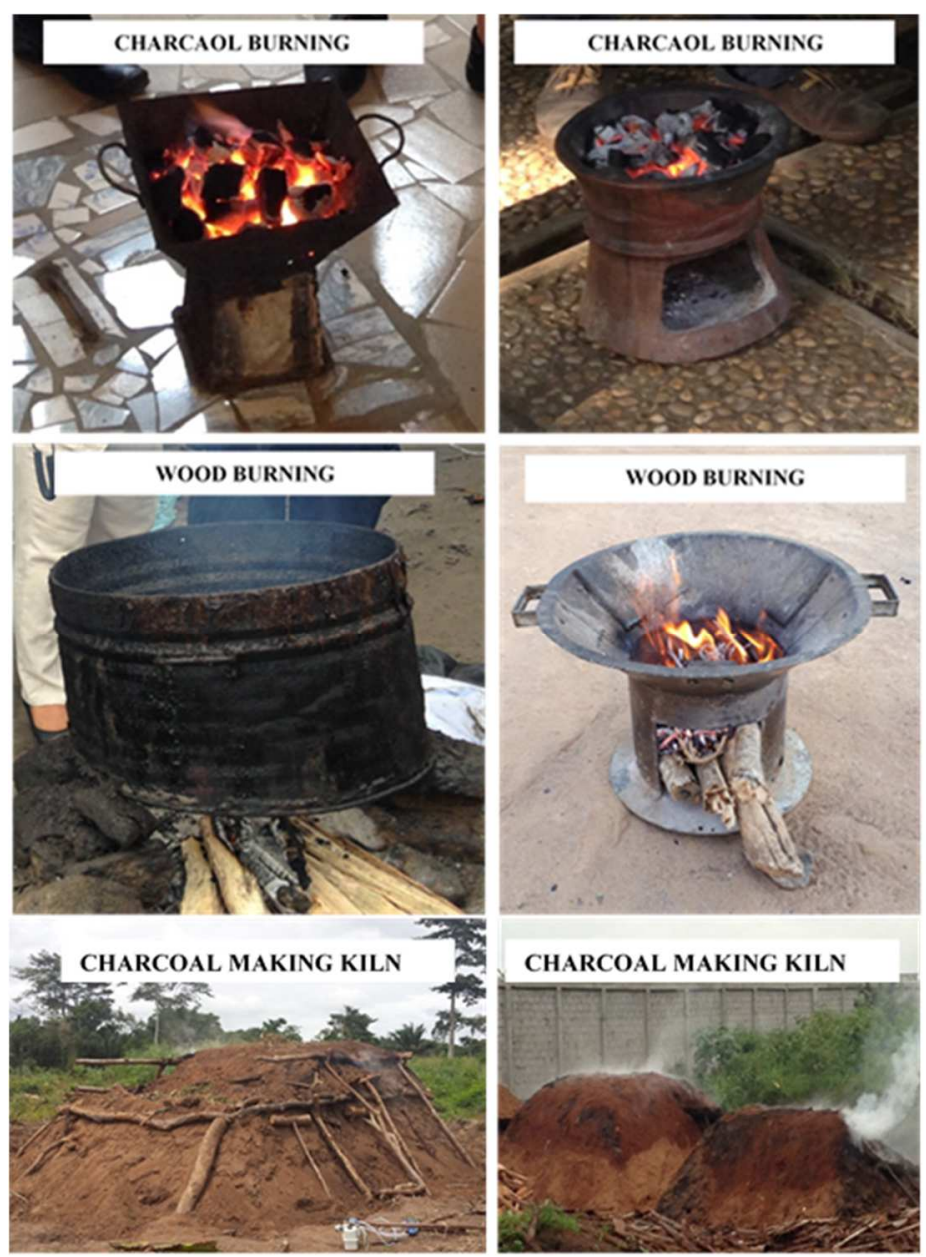

Figure S2: Different stoves used for charcoal, wood cooking fire and charcoal making kiln

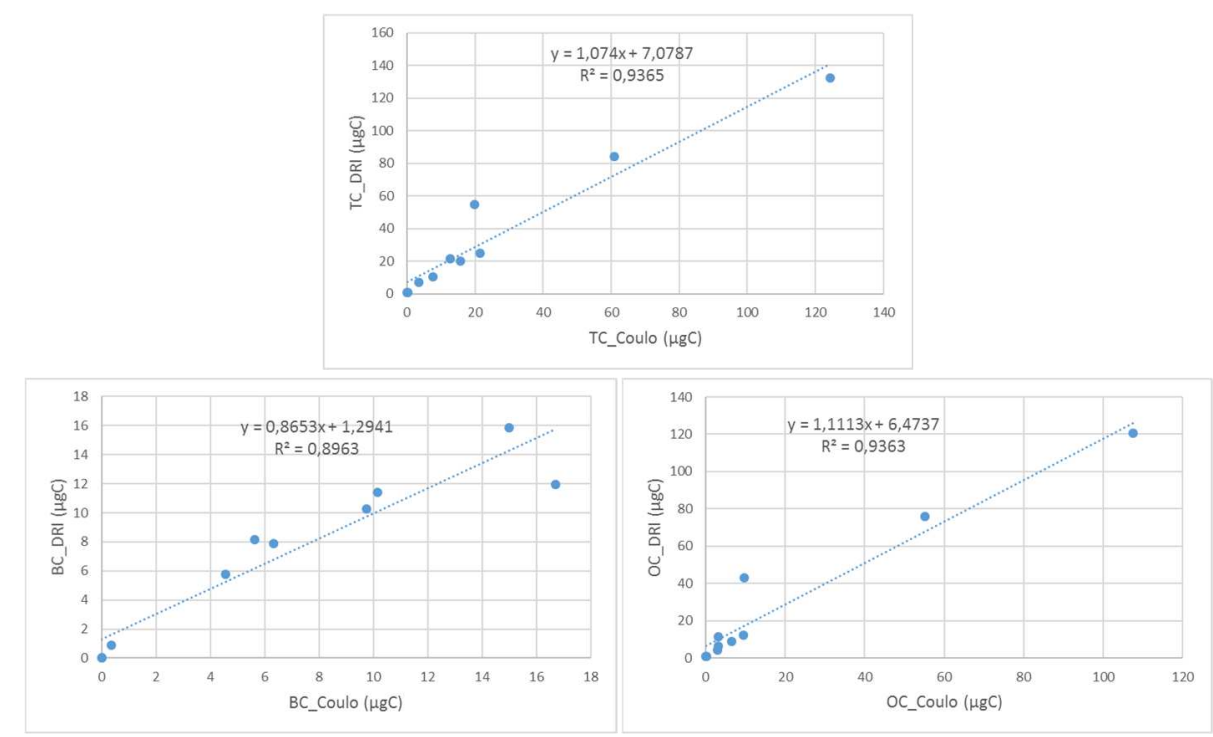

Figure S3: linear regression analysis of each carbonaceous particle values obtained for thermal (coulo) and thermo-optical (DRI) methods 


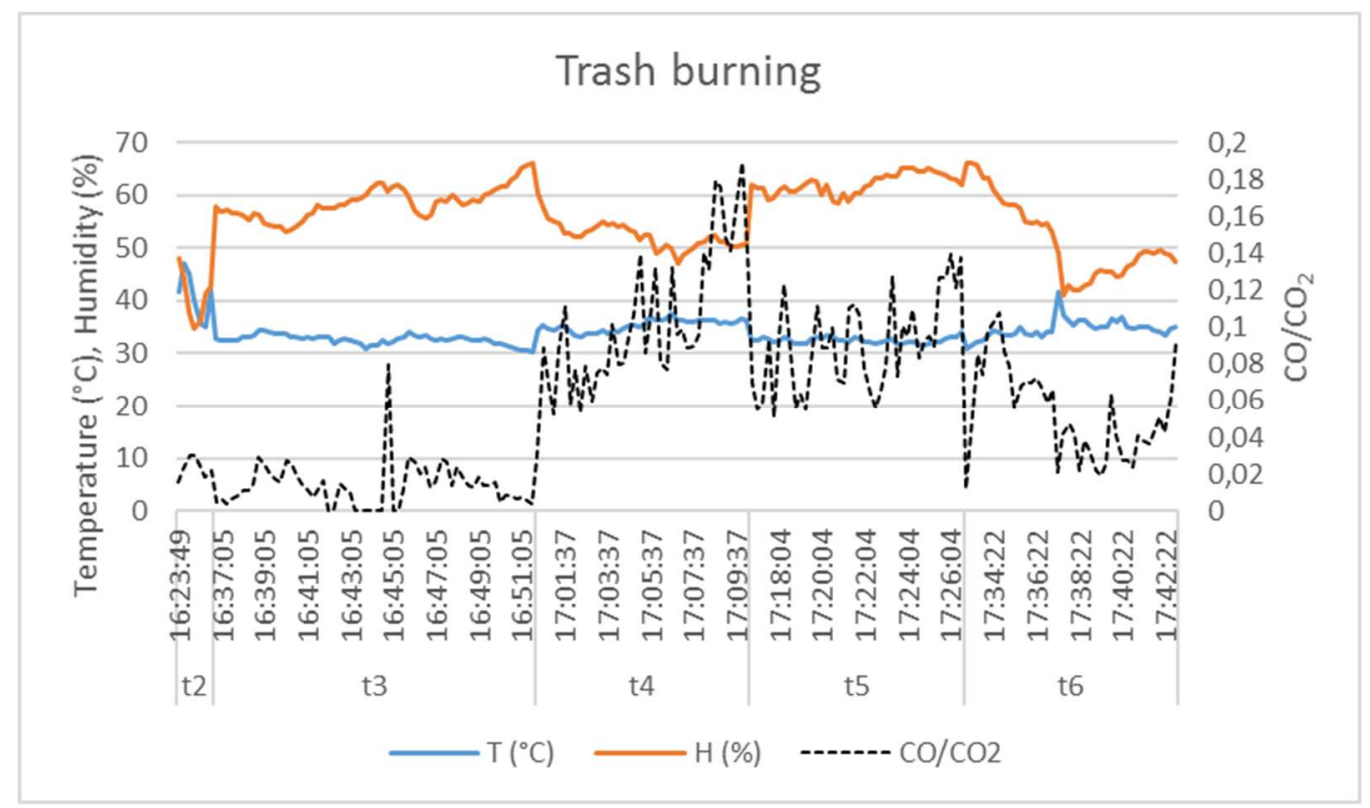

Figure S4: Trash combustion characteristics 


\section{VOC uncertainties and detection limits}

The reproducibility of the method has been evaluated by sampling several cartridges. Details of the test to evaluate the influence of storage conditions are given in Detournay et al (2011). During this study half of the samples were analysed immediately after sampling, while the other half was stored during 30 days before analysis. The results showed excellent relative standard deviations (RSD) for aromatic compounds and alkanes (1\%); and satisfying results were found for terpenes (5\%) and oxygenated compounds $(15 \%)$. This reproducibility gives a good estimation of the measurement uncertainty.

The global uncertainty was also calculate for both methods. Global uncertainty is the square root of the quadratic sum of uncertainties, and it was calculate following the recommendations from French national organization for standardization (AFNOR, 2008). For that, the volume precision, measure precision and measurement accuracy were computed.

$$
u^{2}=\Delta x^{2} \text { volume }+\Delta x^{2} \text { measure }
$$

, were

$$
\Delta x^{2} \text { measure }=\Delta x^{2} \text { precision }+\Delta x^{2} \text { accuracy }
$$

The uncertainty values (U, in percentage \%) for both methods are shown in Table S1 and table S2.

Limit of detection (DL) and limit of quantification (QL) were also estimated and they are shown in Table S1 and S2.

Table S1. Detection limits, quantification limits and global uncertainties for the VOCs analysed at LaMP under GC-MS technique

\begin{tabular}{lrrr}
\hline & $\begin{array}{r}\text { DL } \\
\text { (pptv) }\end{array}$ & $\begin{array}{r}\text { QL } \\
\text { (pptv) }\end{array}$ & $\begin{array}{r}\text { U } \\
\text { (\%) }\end{array}$ \\
\hline Benzene & 10.32 & 51.59 & 13.54 \\
Toluene & 1.76 & 8.81 & 2.53 \\
o-xylene & 2.92 & 14.61 & 4.23 \\
m+p-xylene & 1.29 & 6.43 & 3.10 \\
Ethylbenzene & 3.96 & 19.78 & 3.61 \\
Octane & 4.01 & 20.05 & 5.58 \\
iso-octane & 14.58 & 72.90 & 19.58 \\
Heptane & 2.34 & 11.70 & 9.67 \\
2- methyl pentane & 22.57 & 112.84 & 10.98 \\
Isoprene & 12.19 & 60.96 & 19.63 \\
Limonene & 1.17 & 5.84 & 9.68 \\
及-Pinene & 3.00 & 15.01 & 6.54 \\
a-Pinene & 8.67 & 43.35 & 18.52 \\
1,2,3-trimethylbenzene & 1.10 & 5.49 & 14.33 \\
1,2,4-trimethylbenzene & 2.25 & 11.25 & 6.92 \\
1,3,5-trimethylbenzene & 2.01 & 10.03 & 37.94 \\
\hline
\end{tabular}


Table S2. Detection limits and global uncertainties for the VOCs analysed at IMT Lille with GCFID technique

\begin{tabular}{|c|c|c|c|c|c|}
\hline & $\begin{array}{r}\text { DL } \\
\text { (pptv) }\end{array}$ & $\begin{array}{r}\mathrm{U} \\
(\%)\end{array}$ & & $\begin{array}{r}\text { DL } \\
\text { (pptv) }\end{array}$ & $\begin{array}{r}\mathrm{U} \\
(\%)\end{array}$ \\
\hline hexanal & 3 & 21 & octane & 1 & n.d. \\
\hline heptanal & 4 & 21.2 & nonane & 1 & 9.4 \\
\hline octanal & 2 & 21.4 & decane & 1 & 5.3 \\
\hline nonanal & 4 & 28.5 & undecane & 1 & 4.8 \\
\hline decanal & 2 & 29.3 & dodecane & 1 & 4.9 \\
\hline undecanal & 2 & 21.4 & tridecane & 1 & 32.6 \\
\hline benzaldehyde & 2 & n.d. & tetradecane & $<1$ & 11.5 \\
\hline benzene & 1 & 5.0 & pentadecane & 4 & 10.9 \\
\hline toluene & 2 & 5.4 & hexadecane & 13 & 17.3 \\
\hline ethylbenzene & 1 & 4.0 & $\alpha$-pinene & $<1$ & 4.4 \\
\hline m+p-xylene & $<1$ & 5.7 & camphene & 1 & 5.1 \\
\hline o-xylene & 1 & 3.7 & $\beta$-pinene & 3 & 8.4 \\
\hline $\begin{array}{l}\text { styrene } \\
1,3,5-\end{array}$ & 1 & n.d. & $\alpha$-terpinene & $<1$ & n.d. \\
\hline $\begin{array}{l}\text { trimethylbenzene } \\
1,2,4-\end{array}$ & 1 & n.d. & limonene & 1 & 15.2 \\
\hline $\begin{array}{l}\text { trimethylbenzene } \\
1,2,3-\end{array}$ & 1 & n.d. & $\gamma$-terpinene & 3 & n.d. \\
\hline trimethylbenzene & $<1$ & n.d. & 3-carene & 1 & n.d. \\
\hline pentane & 2 & n.d. & $\alpha$-ocimene & 1 & n.d. \\
\hline 2-methyl pentane & 1 & n.d. & terpinolene & 1 & n.d. \\
\hline hexane & 1 & n.d. & myrcene & 2 & n.d. \\
\hline iso-octane & 1 & n.d. & nopinone & $<1$ & n.d. \\
\hline heptane & 1 & n.d. & & & \\
\hline
\end{tabular}

Table S3: Particle EFs of each vehicle tested and number of filters, VOC tubes sampled

\begin{tabular}{|c|c|c|c|c|c|}
\hline \multirow[t]{2}{*}{ Sources } & \multicolumn{2}{|c|}{$\begin{array}{c}\text { Number of } \\
\text { sampling }\end{array}$} & \multicolumn{3}{|c|}{ EF (g/kg fuel) } \\
\hline & VOCs & Filters & EC & OC & TPM \\
\hline DL_old taxi & \multirow{6}{*}{2} & \multirow{6}{*}{6} & 1.880 & 0.340 & 10.459 \\
\hline DL_old taxi & & & 2.826 & 4.143 & 64.507 \\
\hline DL_old CAR & & & 7.746 & 5.828 & 87.287 \\
\hline DL_old CAR & & & 10.619 & 2.715 & 100.750 \\
\hline DL_old gbaka* & & & 3.062 & 2.889 & 33.143 \\
\hline DL_old gbaka & & & 2.313 & 1.943 & 25.580 \\
\hline Mean old LDDV & & & 4.741 & 2.976 & 53.621 \\
\hline STD & & & 3.270 & 1.712 & 33.019 \\
\hline
\end{tabular}




\begin{tabular}{|c|c|c|c|c|c|}
\hline DL_old Truck & \multirow{4}{*}{4} & \multirow{4}{*}{4} & 4.934 & 3.034 & 57.181 \\
\hline DL_old Truck & & & 5.131 & 7.593 & 84.448 \\
\hline DL_old Bus & & & 0.843 & 1.819 & 17.944 \\
\hline DL_old Bus & & & 2.817 & 2.398 & 28.978 \\
\hline Mean old HDDV & & & 3.431 & 3.711 & 47.138 \\
\hline STD & & & 1.748 & 2.282 & 25.860 \\
\hline DL recent gbaka & \multirow{3}{*}{1} & \multirow{3}{*}{3} & 2.120 & 0.844 & 14.712 \\
\hline DL recent gbaka & & & 1.133 & 0.717 & 7.573 \\
\hline DL recent gbaka & & & 0.519 & 0.250 & 5.098 \\
\hline Mean recent LDDV & & & 1.258 & 0.604 & 9.128 \\
\hline STD & & & 0.659 & 0.255 & 4.076 \\
\hline DL_recent Truck & \multirow{2}{*}{2} & \multirow{2}{*}{2} & 0.339 & 0.570 & 6.103 \\
\hline DL_recent Truck & & & 0.358 & 0.871 & 7.262 \\
\hline Mean recent HDDV & & & 0.348 & 0.721 & 6.682 \\
\hline STD & & & 0.010 & 0.150 & 0.580 \\
\hline Gasoline old car & \multirow{5}{*}{2} & \multirow{5}{*}{5} & 0.518 & 0.770 & 3.992 \\
\hline Gasoline old car & & & 2.383 & 4.241 & 16.264 \\
\hline Gasoline old car & & & 1.626 & 1.466 & 8.639 \\
\hline Gasoline old car & & & 0.363 & 1.660 & 6.312 \\
\hline Gasoline old car & & & 0.277 & 0.888 & 12.965 \\
\hline Mean old LDGV & & & 1.033 & 1.805 & 9.635 \\
\hline STD & & & 0.832 & 1.263 & 4.448 \\
\hline Gasoline new car & \multirow{3}{*}{1} & \multirow{3}{*}{3} & 0.001 & 0.099 & 3.302 \\
\hline Gasoline new car & & & 0.001 & 0.019 & 3.188 \\
\hline Gasoline new car & & & 0.002 & 0.007 & 2.583 \\
\hline Mean recent LDGV & & & 0.001 & 0.042 & 3.024 \\
\hline STD & & & 0.001 & 0.040 & 0.316 \\
\hline TW 4-strokes & \multirow{4}{*}{4} & \multirow{4}{*}{4} & 0.069 & 0.226 & 0.724 \\
\hline TW 4-strokes & & & 0.275 & 0.516 & 3.657 \\
\hline TW 4-strokes & & & 0.039 & 0.509 & 13.108 \\
\hline TW 4-strokes & & & 0.040 & 0.555 & 3.998 \\
\hline Mean recent TW 4T & & & 0.106 & 0.452 & 5.372 \\
\hline STD & & & 0.099 & 0.131 & 4.644 \\
\hline TW 4T vieux & 1 & 1 & 3.665 & 25.467 & 499.872 \\
\hline TW 2-strokes & \multirow{2}{*}{2} & \multirow{2}{*}{2} & 0.845 & 24.587 & 45.283 \\
\hline TW 2-strokes & & & 3.681 & 26.837 & 431.303 \\
\hline Mean recent TW 2T & & & 2.263 & 25.712 & 238.293 \\
\hline STD & & & 1.418 & 1.125 & 193.010 \\
\hline TW $2 \mathrm{~T}$ vieux & 1 & 1 & 3.454 & 124.214 & 883.098 \\
\hline
\end{tabular}

*gbaka is a van used for public transport in Côte d'Ivoire 
Table S4: GEIA VOC species groups (Huang et al., 2017)

\begin{tabular}{|c|c|c|}
\hline Class & VOCs families & VOC species integrated in this study \\
\hline VOC1 & Alkanols (alcohols) & n.d. \\
\hline VOC2 & Ethane & n.d. \\
\hline VOC3 & Propane & n.d. \\
\hline VOC4 & Butanes & n.d. \\
\hline VOC5 & Pentanes & pentane \\
\hline VOC6 & Hexanes and higher alkanes & $\begin{array}{l}\text { 2-methylpentane, 3-methylpentane, hexane, 2,2- } \\
\text { dimethylpentane, 2,4-dimethylpentane, 2,2,3- } \\
\text { trimethylbutane, 3,3-dimethylpentane, cyclohexane, } \\
\text { 2-methylhexane, 2,3-dimethylpentane, iso-octane, } \\
\text { heptane, octane, nonane, decane, undecane, } \\
\text { dodecane, tridecane, tetradecane, pentadecane, } \\
\text { hexadecane }\end{array}$ \\
\hline VOC7 & Ethene (ethylene) & n.d. \\
\hline VOC8 & Propene & n.d. \\
\hline VOC9 & Ethyne (acetylene) & n.d. \\
\hline VOC10 & Isoprenes & isoprene \\
\hline VOC11 & Monoterpenes & $\begin{array}{l}\alpha \text {-pinene, } \beta \text {-pinene, camphene, } 3 \text {-carene, } \alpha \text {-terpinene, } \\
\text { limonene, } \alpha \text {-ocimene, } \gamma \text {-terpinene, terpinolene, } \\
\text { myrcene, camphre, borneol }\end{array}$ \\
\hline VOC12 & Other alk(adi)enes/alkynes (olefines) & n.d. \\
\hline VOC13 & Benzene (benzol) & benzene \\
\hline VOC14 & Methylbenzene (toluene) & toluene \\
\hline VOC15 & Dimethylbenzenes (xylenes) & M+p-xylene, o-xylene, ethylbenzene, \\
\hline VOC16 & Trimethylbenzenes & $\begin{array}{l}\text { 1,2,4-trimethylbenzene, 1,2,3-trimethylbenzene and } \\
\text { 1,3,5-trimethylbenzene }\end{array}$ \\
\hline VOC17 & Other aromatics & Cumene, styrene \\
\hline VOC18 & Esters & n.d. \\
\hline VOC19 & Ethers (alkoxy alkanes) & n.d. \\
\hline VOC20 & Chlorinated hydrocarbons & n.d. \\
\hline VOC21 & Methanal (formaldehyde) & n.d. \\
\hline VOC22 & Other alkanals (aldehydes) & $\begin{array}{l}\text { Benzaldehyde, heptanal, hexanal, octanal, nonanal, } \\
\text { decanal, undecanal }\end{array}$ \\
\hline VOC23 & Alkanones (ketones) & Methylvinylketone, methylethylketone \\
\hline VOC24 & Acids (alkanoic) & n.d. \\
\hline VOC25 & $\begin{array}{l}\text { Other NMVOC (HCFCs, nitriles, } \\
\text { etc.) }\end{array}$ & n.d. \\
\hline
\end{tabular}


Table S5: EFs of emission sources grouped by VOC speciation of GEIA

\begin{tabular}{lccccccccc}
\hline $\begin{array}{c}\text { EF } \\
\text { (g/kg fuel) }\end{array}$ & HDDV & LDDV & LDGV & TW 2T & TW 4T & FW & CHM & WB & CH \\
\hline VOC6 & $1.07 \pm 0.23$ & $0.60 \pm 0.13$ & $2.84 \pm 0.20$ & $1148 \pm 337$ & $22.5 \pm 3.43$ & $2.79 \pm 0.93$ & $5.34 \pm 1.49$ & $16.0 \pm 7.09$ & $1.84 \pm 0.49$ \\
VOC10 & $0.02 \pm 0.02$ & $0.06+0.06$ & $0.41+0.35$ & $28.3 \pm 28.6$ & $1.98 \pm 0.85$ & $1.70 \pm 1.69$ & $0.70 \pm 0.25$ & $2.67 \pm 4.33$ & $0.20 \pm 0.21$ \\
VOC11 & $0.17 \pm 0.04$ & $0.03+0.00$ & $0.06+0.02$ & $53.2 \pm 20.5$ & $0.36 \pm 0.07$ & $1.94 \pm 0.64$ & $0.37 \pm 0.11$ & $69.5 \pm 26.3$ & $2.03 \pm 1.11$ \\
VOC12 & $0.00 \pm 0.00$ & $0.21+0.02$ & $2.09+1.02$ & $87.9 \pm 106$ & $0.10 \pm 0.05$ & $0.00 \pm 0.00$ & $0.12 \pm 0.12$ & $1.58 \pm 2.14$ & $0.27 \pm 0.42$ \\
VOC13 & $0.68 \pm 0.27$ & $5.57+2.80$ & $4.78+0.37$ & $380 \pm 279$ & $32.0 \pm 8.56$ & $2.00 \pm 1.99$ & $4.20 \pm 0.12$ & $19.1 \pm 19.0$ & $8.64 \pm 11.9$ \\
VOC14 & $0.58 \pm 0.18$ & $3.10+1.80$ & $34.8+18.6$ & $1135 \pm 830$ & $95.1 \pm 32.2$ & $1.53 \pm 1.52$ & $4.64 \pm 2.02$ & $35.5 \pm 45.6$ & $3.60 \pm 4.30$ \\
VOC15 & $1.01 \pm 0.20$ & $1.62+0.25$ & $93.5+2.25$ & $2127 \pm 673$ & $81.3 \pm 15.2$ & $1.59 \pm 0.79$ & $3.37 \pm 0.98$ & $5.86 \pm 4.52$ & $2.69 \pm 1.26$ \\
VOC16 & $2.13 \pm 0.52$ & $2.96+0.21$ & $51.2+0.59$ & $1916 \pm 437$ & $46.3 \pm 7.00$ & $0.78 \pm 0.26$ & $2.89 \pm 0.34$ & $4.35 \pm 1.92$ & $7.87 \pm 4.30$ \\
VOC17 & $0.25 \pm 0.11$ & $0.82+0.37$ & $15.6+3.15$ & $815 \pm 591$ & $40.6 \pm 16.2$ & $0.24 \pm 0.24$ & $0.81 \pm 0.25$ & $27.9 \pm 34.3$ & $1.74 \pm 2.07$ \\
\hline
\end{tabular}

Table S6: EFs of emission sources grouped by VOC speciation of GEIA for 50 compounds

\begin{tabular}{lrrrrr}
\hline $\begin{array}{c}\text { EF } \\
\text { (g/kg fuel) }\end{array}$ & HDDV & TW 2T & FW & CHM & CH \\
\hline VOC5 & 0.07 & 331.2 & 0.15 & 1.03 & 0.25 \\
VOC6 & 115.3 & 6659 & 10.4 & 23.8 & 10.8 \\
VOC10 & 0.34 & 46.10 & 3.39 & 0.87 & 0.09 \\
VOC11 & 19.4 & 574.7 & 4.83 & 3.81 & 1.06 \\
VOC12 & 0.92 & 187.6 & 0.00 & 0.45 & 0.12 \\
VOC13 & 20.8 & 617.7 & 3.99 & 8.26 & 3.56 \\
VOC14 & 9.79 & 1841 & 3.05 & 12.7 & 1.56 \\
VOC15 & 12.0 & 3220 & 3.17 & 10.2 & 1.18 \\
VOC16 & 22.5 & 2847 & 1.55 & 5.68 & 3.61 \\
VOC17 & 4.42 & 1953 & 1.30 & 3.72 & 1.15 \\
VOC22 & 33.4 & 665.7 & 2.26 & 17.3 & 3.53 \\
VOC23 & 1.14 & 10.69 & 0.44 & 15.2 & 0.36 \\
IVOCs & 80.32 & 95.07 & 1.17 & 6.32 & 0.73 \\
\hline
\end{tabular}


Table S7: VOC-EFs from diesel and gasoline light- and heavy-duty vehicles and wood burning in this study and literature

\begin{tabular}{|c|c|c|c|c|c|c|c|c|c|c|c|c|c|c|c|}
\hline \multirow{3}{*}{$\begin{array}{l}\text { EF } \\
\text { Unit } \\
\text { Reference } \\
\text { heptane }\end{array}$} & \multirow{2}{*}{\multicolumn{2}{|c|}{$\begin{array}{l}\text { HDDV std } \\
\text { (g/kgdm) } \\
\text { This study }\end{array}$}} & \multirow{3}{*}{$\begin{array}{l}\text { LDDV } \\
\text { (g/kgdm) } \\
0.18\end{array}$} & \multirow{3}{*}{$\begin{array}{l}\text { std } \\
0.13\end{array}$} & \multirow{3}{*}{$\begin{array}{l}\begin{array}{l}\text { LDGV } \\
\text { (g/kgdm) }\end{array} \\
0.44\end{array}$} & \multirow{3}{*}{$\begin{array}{l}\text { std } \\
0.15\end{array}$} & \multirow{3}{*}{$\begin{array}{l}\text { FW } \\
(\mathrm{g} / \mathrm{kgdm}) \\
0.04\end{array}$} & \multirow{3}{*}{$\begin{array}{l}\text { std } \\
0.04\end{array}$} & \multirow{2}{*}{$\begin{array}{l}\text { Gasoline_HDLI std } \\
\text { g/L } \\
\text { Gentner et al., } 2016\end{array}$} & \multirow{3}{*}{$\begin{array}{l}\text { std } \\
g / L\end{array}$} & \multirow{4}{*}{$\begin{array}{l}\text { std } \\
0.003 \\
\end{array}$} & \multirow{2}{*}{\multicolumn{2}{|c|}{$\begin{array}{l}\text { WB maximum std } \\
\text { g/L } \\
\text { Evtyugina et al, } 2015\end{array}$}} & \multirow{2}{*}{\multicolumn{2}{|c|}{$\begin{array}{l}\text { WB maximum std } \\
\mathrm{g} / \mathrm{kg} \\
\text { McDonaldet al., } 2000\end{array}$}} \\
\hline & & & & & & & & & & & & & & & \\
\hline & 0.23 & 0.16 & & & & & & & 0.042 & & & 0.019 & 0.002 & 0.005 & 0.003 \\
\hline octane & 0.74 & 0.46 & 0.21 & 0.1 & 2.36 & 0.4 & 2.38 & 2.3 & 0.020 & 10.004 & & 0.005 & 0.001 & 0.004 & 0.003 \\
\hline iso-octane & 0.09 & 0.07 & 0.21 & 0.15 & 0.04 & 0.03 & 0.37 & 0.6 & 0.067 & 20.000 & 0.000 & & & & \\
\hline benzene & 0.68 & 0.27 & 5.6 & 2.8 & 4.78 & 0.4 & 2 & 1.98 & 0.227 & 20.006 & 0.005 & 0.901 & 0.131 & 0.312 & 0.083 \\
\hline toluene & 0.58 & 0.17 & 3.1 & 1.8 & 34.7 & 18.6 & 1.53 & 1.52 & 0.226 & 40.022 & 0.013 & 0.326 & 0.047 & 0.142 & 0.004 \\
\hline$m+p-x y l e n e$ & 0.7 & 0.26 & 1.06 & 0.27 & 63.4 & 1.07 & 1.43 & 1.42 & 0.079 & 40.007 & 0.004 & 0.040 & 0.007 & 0.041 & 0.012 \\
\hline o-xylene & 0.32 & 0.15 & 0.56 & 0.22 & 30.1 & 3.43 & 0.16 & 0.47 & 0.057 & 40.004 & 0.003 & 0.027 & 0.002 & & \\
\hline ethylbenzene & 0.25 & 0.11 & 0.82 & 0.37 & 15.6 & 3.15 & 0.24 & 2.07 & 0.033 & 40.009 & 0.005 & 0.053 & 0.006 & & \\
\hline 135-TMB & 0.33 & 0.21 & 0.52 & 0.14 & 10.8 & 0.56 & 0.07 & 0.02 & 0.019 & 00.015 & 0.008 & 0.001 & 0.000 & & \\
\hline 124-TMB & 1.29 & 0.96 & 1.73 & 0.34 & 34.6 & 0.84 & 0.06 & 0.08 & 0.093 & 10.046 & 0.024 & & & & \\
\hline 123-TMB & 0.5 & 0.4 & 0.71 & 0.14 & 5.84 & 0.36 & 0.65 & 12.7 & & & & & & & \\
\hline isoprene & 0.02 & 0.02 & 0.06 & 0.06 & 0.41 & 0.35 & 1.69 & 0.2 & & & & 0.111 & 0.016 & 0.027 & 0.013 \\
\hline limonene & 0.07 & 0.06 & 0.02 & 0.01 & 0 & 0 & 1.91 & 1.87 & & & & 0.001 & 0.001 & 0.018 & 0.027 \\
\hline a-pinene & 0.04 & 0.02 & 0 & 0 & 0.01 & 0.01 & 0 & 1.5 & & & & 0.009 & 0.004 & 0.004 & 0.004 \\
\hline b-pinene & 0.06 & 0.05 & 0.01 & 0 & 0.05 & 0.03 & 0.03 & 1.72 & & & & & & & \\
\hline
\end{tabular}

\section{References}

Huang, G., Brook, R., Crippa, M., Janssens-Maenhout, G., Schieberle, C., Dore, C., Guizzardi, D., Muntean, M., Schaaf, E. and Friedrich, R.: Speciation of anthropogenic emissions of non-methane volatile organic compounds: A global gridded data set for 1970-2012, Atmos. Chem. Phys., 17(12), 7683-7701, doi:10.5194/acp-17-7683-2017, 2017.

Detournay, A., Sauvage, S., Locoge, N., Gaudion, V., Leonardis, T., Fronval, I., Kaluzny, P. and Galloo, J.-C.: Development of a sampling method for the simultaneous monitoring of straight-chain alkanes, straight-chain saturated carbonyl compounds and monoterpenes in remote areas, J. Environ. Monit., 13(4), 983, doi:10.1039/c0em00354a, 2011.

AFNOR: Guide pratique d'utilisation pour l'estimation de l'incertitude de mesure des concentrations en polluants dans l'air ambiant - Partie 5 : Estimation des incertitudes de mesurages de benzène réalisés sur site par pompage suivis d'une désorption thermique et d'une analyse chromatographique en phase gazeuse, 2008. 